ALLEPUZ GARCÍA, Pablo (2016): "El estudio arquitectónico 'lapanadería' (20032013) desde la filosofía de Gilles Deleuze y Félix Guattari". Ángulo Recto. Revista de estudios sobre la ciudad como espacio plural, vol. 8, núm. 1-2, pp. 5-25. ISSN: 1989-4015.

http://dx.doi.org/10.5209/rev_ANRE.2016.v8.n1.53346

\title{
El estudio arquitectónico "lapanadería" (2003-2013) desde la filosofía de Gilles Deleuze y Félix Guattari
}

\author{
Pablo Allepuz García \\ Departamento de Historia del Arte, Arqueología y Música \\ Universidad de Córdoba \\ pabloallepuz@gmail.com
}

Recibido: $12 / 02 / 2016$

Aceptado: $13 / 07 / 2016$

\section{Resumen}

Entre los años 2003 y 2013 el estudio arquitectónico lapanadería mantuvo una importante línea de investigación y praxis en torno a una propuesta de vivienda incremental en la que es posible identificar tres niveles consecutivos: la casa más o menos, algo así como un barrio más o menos y una plataforma web de procesos colectivos de vivienda llamada Más que una casa. Las teorías estético-políticas de Gilles Deleuze y Félix Guattari, y en especial Las tres ecologías (1989) de este último, posibilitarán un análisis crítico de cada nivel y una exégesis global de la iniciativa.

Palabras clave: lapanadería, Deleuze, Guattari, casa más o menos, ecología.

Title: The Architectural Studio "lapanadería" (2003-2013) from Gilles Deleuze and Félix Guattari's Philosophy

\section{Abstract}

Between 2003 and 2013 the architectural studio lapanadería ["thebakery"] developed an important line of research and practice around a proposal for incremental housing in which it is possible to identify three consecutive levels: the more-or-less house, such thing as a more-or-less neighbourhood and a web platform for collective processes of housing called Más que una casa ["more than a house"]. Gilles Deleuze and Félix Guattari's aestheticpolitical theories, especially The Three Ecologies (1989) of the latter, will enable a critical analysis of each level and a global exegesis of the initiative.

Keywords: lapanadería, Deleuze, Guattari, more-or-less house, ecology.

\section{Índice}

1. La vivienda incremental y el pensamiento de Deleuze y Guattari

2. Casa más o menos

3. Barrio más o menos

4. Más que una casa; ¿hacia una ciudad más o menos?

5. ¿Más, o menos? Últimas consideraciones 
ALLEPUZ GARCÍA, Pablo (2016): "El estudio arquitectónico 'lapanadería' (20032013) desde la filosofía de Gilles Deleuze y Félix Guattari". Ángulo Recto. Revista de estudios sobre la ciudad como espacio plural, vol. 8, núm. 1-2, pp. 5-25. ISSN: 1989-4015.

http://dx.doi.org/10.5209/rev_ANRE.2016.v8.n1.53346

\section{La vivienda incremental y el pensamiento de Deleuze y Guattari}

Afirma Pedro Lorenzo (vid. Lapanadería y Lorenzo 2010) que el contexto político ejerce un peso fundamental sobre el ejercicio arquitectónico en al menos cuatro grados de control: el primero, el estado neoliberal o globalizado, el modelo dominante, en el que priman los intereses y en el que el usuario debe aceptar lo que se le plantee; el segundo, el gobierno social, dispone de políticas de observación del usuario, como la Vivienda de Protección Oficial; el tercero podría generalizarse como gobierno facilitador, pues tiene mecanismos de solución de problemas en los cuales participa el usuario (son habituales en Latinoamérica, a través de suelo equipado, vivienda progresiva, vivienda semilla, vivienda evolutiva, planes techo...); el cuarto, casi utópico, es el gobierno participativo que llama a la cogestión al usuario. Si hubiera que situar a España dentro de esta clasificación, la casuística de la vivienda podría alcanzar un segundo grado, aunque condicionado por un macrosistema económico general del primer grado, dentro de lo que Guattari denomina Capitalismo Mundial Integrado (en adelante CMI).

El espacio, la habitación, la ciudad, el territorio, se han desvelado, como un lugar privilegiado de la lucha -conocimiento y acción-, aquí y ahora, por el control sobre nuestras propias vidas, de la construcción de la autonomía. Foucault lo enunció como las tácticas locales del hábitat. (Pérez de Lama 2002-2003: 188)

Para Guattari, la evolución de la humanidad se puede resumir en tres etapas: la cristiandad europea, la desterritorialización capitalista y la informatización planetaria; fase esta última en que se inserta el CMI: mediante el dominio de los ritornelos existenciales, este serializa y homogeneiza a la sociedad, laminando su subjetividad (1996: 47-49). Contra tales prácticas, y junto a un Negri que ya había pensado las "máquinas ecológicas" (Moreno García 2000-2001: 146-147), Guattari opone y propone "nuevos espacios de libertad", una reterritorialización y una resubjetivación.

[...] las tres ecologías [...] dependen de lo que yo he llamado una heterogénesis, es decir, de procesos continuos de resingularización. Los individuos han de devenir a la vez solidarios y cada vez más diferentes. (Lo mismo sucede con la resingularización de las escuelas, de los ayuntamientos, del urbanismo, etc.). (Guattari 1996: 79)

En ¿Qué es la filosofía?, Deleuze y Guattari (1997: 202-220) exponen que el cosmos sale del caos mediante la introducción del orden, dando lugar a la complejidad; así, la ciencia, la filosofía y el 
ALLEPUZ GARCÍA, Pablo (2016): "El estudio arquitectónico 'lapanadería' (20032013) desde la filosofía de Gilles Deleuze y Félix Guattari". Ángulo Recto. Revista de estudios sobre la ciudad como espacio plural, vol. 8, núm. 1-2, pp. 5-25. ISSN: 1989-4015.

http://dx.doi.org/10.5209/rev_ANRE.2016.v8.n1.53346

arte deben combinarse para obtener el pensamiento. Tal vez donde mejor expresada se encuentre esta conjunción, así como la de los propios autores, sea en Las tres ecologías (Guattari 1996), que pretendía superar el más o menos manido ambientalismo (1996: 50) para generar algo mucho más complejo y funcional en torno a tres conceptos vertebradores, casi trasuntos de aquellas tres partes del pensamiento: la ecología medioambiental o técnica, procedente de dicho ambientalismo manipulado por el lenguaje político y los massmedia; la ecología social, que se inserta en nuestras relaciones con los demás, y la ecología mental, que atiende a la relación de cada uno con su propia persona y su propio yo. En suma, la plasmación de la ecosofía, entendida en la vertiente etimológica más amplia del oikos, debería contribuir a rearticular de forma transversal lo político y lo privado, lo social, lo ambiental y lo mental (cfr. Martínez 2008: 171).

Deleuze y Guattari compaginan dos visiones del mundo, una estratificada, extensiva, que comparten con autores como Popper, Ferrater, Bunge, etc., y que se inspira en un modelo geológico y un modelo lingüístico a la vez, y otra intensiva que se sitúa en la estela de la teoría espinosiana de la relación entre la Substancia y los modos. Los estratos son siempre plurales y unos sirven de sustratos a los otros. [...] La relación entre los estratos la expresa Guattari con la noción, tomada de G. Bateson, de double bind (doble pinza). [...] A cada articulación le corresponde un tipo de multiplicidad, una molecular, elemental y flexible, la otra molar, orgánica y rígida. (Martínez 2008: 66-67)

Aun cuando Deleuze ha escrito mucho sobre literatura, pintura, cine, performance... y, en cambio, apenas unas pocas líneas sobre arquitectura, el desarrollo de su pensamiento se construye siempre a partir de metáforas espaciales como el plano de inmanencia, el pliegue, las mesetas, la (des- o re-) territorialización.... Y si en Lógica de la sensación afirmaba que la cabeza es pieza de carne y lugar privilegiado del devenir-animal (Deleuze 2009: 29-40), después confirmaría, junto a Guattari, que "el arte no empieza con la carne, sino con la casa; por este motivo la arquitectura es la primera de las artes" (1997: 188).

La cuestión de la vivienda social había entrado en el debate arquitectónico a comienzos del siglo $X X$, máxime tras cada una de las dos Guerras Mundiales, y pronto se convirtió en uno de los puntos básicos del Movimiento Moderno, en torno al modelo de machine à habiter propuesto por Le Corbusier, y de los Congresos Internacionales de Arquitectura Moderna. A partir de la década de 1950, sin embargo, las reflexiones sobre la ciudad en general y la 
ALLEPUZ GARCÍA, Pablo (2016): "El estudio arquitectónico 'lapanadería' (20032013) desde la filosofía de Gilles Deleuze y Félix Guattari". Ángulo Recto. Revista de estudios sobre la ciudad como espacio plural, vol. 8, núm. 1-2, pp. 5-25. ISSN: 1989-4015.

http://dx.doi.org/10.5209/rev_ANRE.2016.v8.n1.53346

casa en particular tomaron una dirección bien distinta al prurito de funcionalidad racionalista: primero fue el pensamiento de Martin Heidegger y su "no habitamos porque hemos construido, sino que construimos porque habitamos, porque somos habitantes" (Heidegger 2007: 212), apuntando con ello la necesidad de una arquitectura que partiera del habitar peculiar de sus habitantes en lugar de adaptarse estos a las soluciones predeterminadas por los arquitectos; poco después, en el CIAM IX (1953), el cisma interno se puso de manifiesto en la oposición de la Carta de habitación frente a la previa Carta de Atenas (1933/1942) y, asimismo, por el nacimiento del Team X; más tarde vendrían el Manifiesto del moho contra el racionalismo en la arquitectura (1958) de Hundertwasser, el urbanismo unitario de la Internacional Situacionista o las experiencias individuales de Constant.

A raíz del mayo francés de 1968 , en el que se encontraban latentes muchas de estas preocupaciones, el pensamiento de Deleuze y Guattari sería clave para la configuración de un nuevo imaginario urbano. Lo relata de forma somera García Vázquez (cfr. 2004: 130147) mediante el paradigma de la "ciudad de los cuerpos", por supuesto de cuerpos sin órganos pero con umbrales o niveles, caracterizada por los procesos de inspiración, evaporación y transmisión de fluidos, y en cuyo seno los "monumentos-máquinas sociales" fueran transformados en "acontecimientos-máquinas deseantes".

El cuerpo sin órganos se opone menos a los órganos que a esa organización de los órganos que se llama organismo. Es un cuerpo intenso, intensivo. [...] Una onda de amplitud variable recorre el cuerpo sin órganos: traza en él zonas y niveles según las variaciones de su amplitud. Una sensación aparece con el encuentro de la onda en tal nivel y de fuerzas exteriores. Así pues, un órgano estará determinado por este encuentro, pero un órgano provisional, que sólo dura lo que duran el paso de la onda y la acción de la fuerza, y que se desplazará para posarse en otra parte. (Deleuze 2009: 51, 53)

En clave de revolución molecular y de intensidad diferencial también pueden entenderse, por ejemplo, las "recetas urbanas" del sevillano Santiago Cirugeda, verdaderos exponentes de los agencements o "componendas" que Deleuze y Guattari adelantaron en Kafka. Por una literatura menor y que ya a partir de Mil Mesetas sustituyeron por completo a las "máquinas-deseantes" (Pardo 2014: 359-378); funcionarían, por tanto, como estrategias micropolíticas contra lo que Francesc Muñoz ha dado en Ilamar "urbanalización", esto es, el "proceso absoluto de simplificación urbana, de pérdida de 
ALLEPUZ GARCÍA, Pablo (2016): "El estudio arquitectónico 'lapanadería' (20032013) desde la filosofía de Gilles Deleuze y Félix Guattari". Ángulo Recto. Revista de estudios sobre la ciudad como espacio plural, vol. 8, núm. 1-2, pp. 5-25. ISSN: 1989-4015.

http://dx.doi.org/10.5209/rev_ANRE.2016.v8.n1.53346

la diversidad y la complejidad que puede y debe contener la ciudad" (Muñoz 2008: 199).

Pero, sin duda, el proyecto que mejor se adapta al pensamiento de Deleuze y Guattari, y a la "égida ecosófica" (Guattari 1996: 30), es el de la "vivienda incremental": el colectivo ELEMENTAL y su fundador Alejandro Aravena, quien en 2016 ha recibido el Premio Pritzker y la dirección artística de la $15^{a}$ Bienal de Arquitectura de Venecia, partieron de una idea de "ciudad como fuente de equidad" (Aravena 2008: 116-117) para llegar al desarrollo de la "casa a medio terminar", realizada en los proyectos de "Quinta Monroy" (2007) y "Villa Verde" (2013) (Aravena y Iacobelli 2012); consistía en rentabilizar el suelo tomando la decisión de, en lugar de optar por una vivienda pequeña, construir la mitad de una más grande, con la posibilidad ulterior de ampliarla hasta la máxima capacidad del espacio dado.

Estos antecedentes históricos y filosóficos eran bien conocidos en la Escuela Técnica Superior de Arquitectura de la Universidad de Sevilla, como lo atestiguan las lecciones y los artículos de José Ramón Moreno García (2000-2001), David Moreno Rangel (20022003) o José Pérez de Lama (2002-2003); no por casualidad, todos publicados en la revista del Departamento de Historia, Teoría y Composición Arquitectónicas de la misma institución. Si a ello añadimos la metáfora del hojaldre presente en García Vázquez (2004) y en la reseña de su compañero Parra Bañón (2004-2005), dentro de idéntico contexto, quizá sirva de explicación para las nuevas hornadas de arquitectos sevillanos.

El estudio arquitectónico lapanadería surgió en 2003 como una iniciativa de Eva Morales Soler, David Cañavate Cazorla y Rubén Alonso Mallén, junto con otros colaboradores, y desapareció como tal a finales de 2013; una simple comparación de fechas revela que sus propuestas son, en buena medida, paralelas a las del prestigioso Aravena. Durante sus años de actividad llevaron a cabo un gran número de proyectos, perfectamente expuestos y documentados en su página web (vid. Lapanadería 2013a, 2013b), de entre los cuales se analizarán tres: la casa más o menos, algo así como un barrio más o menos y la plataforma web de procesos colectivos de vivienda denominado Más que una casa; en ninguno de ellos, por cierto, se explicita la posible vinculación con Deleuze y Guattari (cfr. Lapanadería 2010).

El pan como la arquitectura que nos gusta, aquella que se dirige a lo básico y esencial, aquella moldeable y adaptable... y asimismo el espacio físico de lapanadería se transforma, mengua, se diluye o se expande en función de los tiempos, los colaboradores y las 
ALLEPUZ GARCÍA, Pablo (2016): "El estudio arquitectónico 'lapanadería' (20032013) desde la filosofía de Gilles Deleuze y Félix Guattari". Ángulo Recto. Revista de estudios sobre la ciudad como espacio plural, vol. 8, núm. 1-2, pp. 5-25. ISSN: 1989-4015.

http://dx.doi.org/10.5209/rev_ANRE.2016.v8.n1.53346

coyunturas... mientras tanto su espacio virtual se ramifica y crece... (Lapanadería 2012: 1)

\section{Casa más o menos}

¿Cómo sería la forma de una arquitectura que aparentemente no haya envejecido y que sin embargo linde simultáneamente con épocas distintas? [se preguntaba Moreno García en su conferencia "Estancias"]. Será una arquitectura de la modificación. Una arquitectura que oscila entre lo global y lo particular, que funda en cada obra sus propias reglas, como pertenencia frente al aislamiento ideal y que establece puentes con el caos que le rodea. Una arquitectura de reborde. El borde es donde se plantean siempre los más desconcertantes problemas de topología. (cfr. Moreno Rangel 2002-2003: 124)

La casa más o menos, premiada en diversos ámbitos, perseguía dar respuesta a las difíciles condiciones de acceso a la vivienda para el común de la población y, más concretamente, para las minorías de jóvenes. La consideración de la vivienda como producto completo y bien de especulación ha sido utilizada por el mercado hasta la insostenibilidad, negando el derecho constitucional a la vivienda. Así pues, se hace necesario plantear vías alternativas de desarrollo que no mantengan el lucro como principal objetivo $y$, de paso, que se adapten a las posibilidades y necesidades del habitante en cada momento (figuras 1 y 2$)^{1}$.

El concepto de vivienda como proceso se fundamenta en la necesidad de adecuar la vivienda a los modos de vida y el medio ambiente actuales. Partiendo de que estos modos de vida son cambiantes y que las necesidades habitacionales y ambientales futuras no se pueden prever, se plantea concebir la vivienda como un proceso y no como un objeto acabado y ligado a la inversión, donde se puedan llevar a cabo transformaciones espaciales y tecnológicas en una relación dinámica entre formas de vida y espacios habitables, como un sistema complejo, como un socioecosistema. (Lapanadería 2010: 40)

\footnotetext{
${ }^{1}$ Enrique Ortiz Flores habla sobre la vivienda como proceso (vid. Lapanadería y Ortiz 2013a).
} 
ALLEPUZ GARCÍA, Pablo (2016): "El estudio arquitectónico 'lapanadería' (20032013) desde la filosofía de Gilles Deleuze y Félix Guattari". Ángulo Recto. Revista de estudios sobre la ciudad como espacio plural, vol. 8, núm. 1-2, pp. 5-25. ISSN: 1989-4015.

http://dx.doi.org/10.5209/rev_ANRE.2016.v8.n1.53346
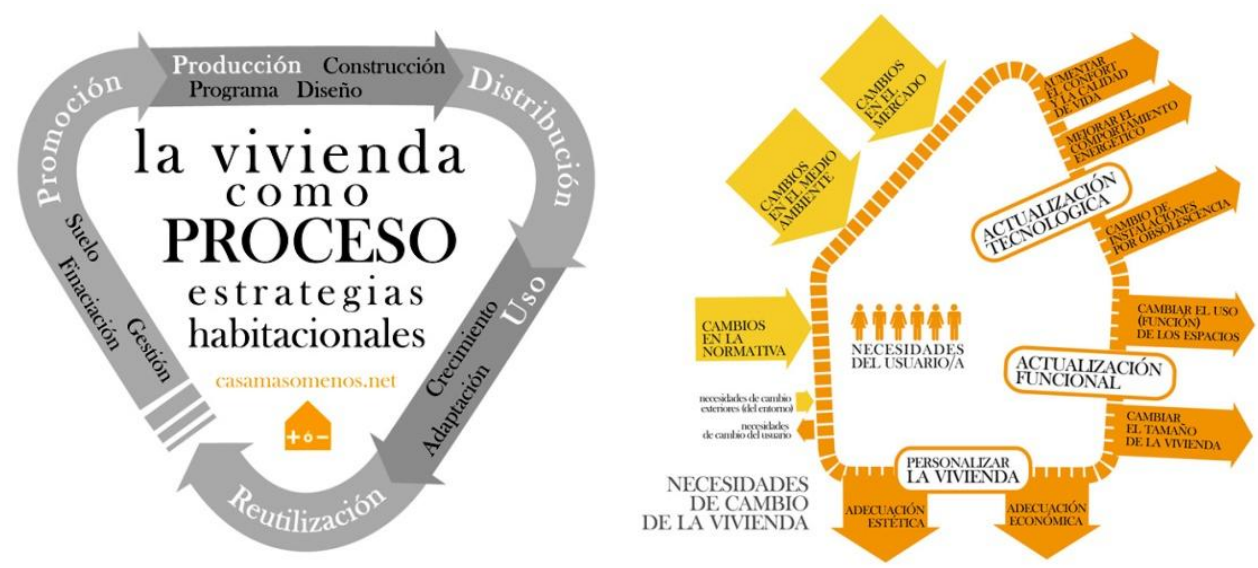

Figuras 1 y 2. Diagramas sobre la vivienda como proceso.

Fuente: Lapanadería.

El concepto procesual, de flexibilidad en el producto, va implícito en las prácticas de cohabitación generales (vid. Lapanadería y Andrade 2010), aunque como una práctica a posteriori y nunca como concepto generador. Esa es precisamente la característica distintiva, que entronca en cierta manera con el concepto de espacio liso frente a espacio estriado (Deleuze y Guattari 2010: 483-506) y más directamente con el del devenir deleuziano:

[...] devenir es, a partir de las formas que se tienen, del sujeto que se es, de los órganos que se poseen o de las funciones que se realizan, extraer partículas entre las que se instauran relaciones de movimiento y reposo, de velocidad y de lentitud, lo más próximas posibles a lo que se está deviniendo y gracias a las cuales se deviene. En ese sentido, el devenir es el proceso del deseo. (Deleuze y Guattari 2010: 275)

De igual manera, Deleuze y Guattari consideran que es en los márgenes donde se pueden encontrar los puntos de ruptura en las estructuras sociales y la irrupción de nuevas formas colectivas de desear, es decir, una experimentación social, pues lo marginal se define siempre respecto a una pretendida normalidad $y$, además, sugiere la necesidad de integración y recuperación. Lapanadería justifica la marginalidad desde un punto de vista más pragmático, argumentando el precio del suelo más favorable y la apertura más o menos forzada de los grupos sociales debido al contexto de crisis económica, pero el contenido de fondo es el mismo

El devenir minoritario es una cuestión de política, de micropolítica activa, de actuación de la potencia. De despliegue de un sujeto desterritorializado a través de un medio también él mismo desterritorializado. Lo minoritario nunca se puede dar como un sujeto 
ALLEPUZ GARCÍA, Pablo (2016): "El estudio arquitectónico 'lapanadería' (20032013) desde la filosofía de Gilles Deleuze y Félix Guattari". Ángulo Recto. Revista de estudios sobre la ciudad como espacio plural, vol. 8, núm. 1-2, pp. 5-25. ISSN: 1989-4015.

http://dx.doi.org/10.5209/rev_ANRE.2016.v8.n1.53346

cerrado y concluso; tampoco se basa en un territorio definido sino que es el producto del descentramiento de un sujeto (que como tal siempre es mayoritario) y se abre hacia una tierra que es el producto de la desterritorialización de un territorio. Por ello, lo minoritario siempre se da como devenir, nunca como estado o situación. (Martínez 2008: 89)

Y todo ello, como podrá comprenderse, supone un continuo proceso de re-territorialización y re-subjetivación en consonancia con lo expuesto por Guattari:

En cada núcleo existencial parcial, las praxis ecológicas se esforzarán en localizar los vectores potenciales de subjetivación y singularización. Generalmente se trata de algo que se opone al orden "normal" de las cosas, una repetición contrariante, un elemento intensivo que reclama otras intensidades a fin de componer otras configuraciones existenciales. Estos vectores disidentes están relativamente despojados de sus funciones de denotación y de significación, para actuar en tanto que materiales existenciales descorporeizados. (Guattari 1996: 37)

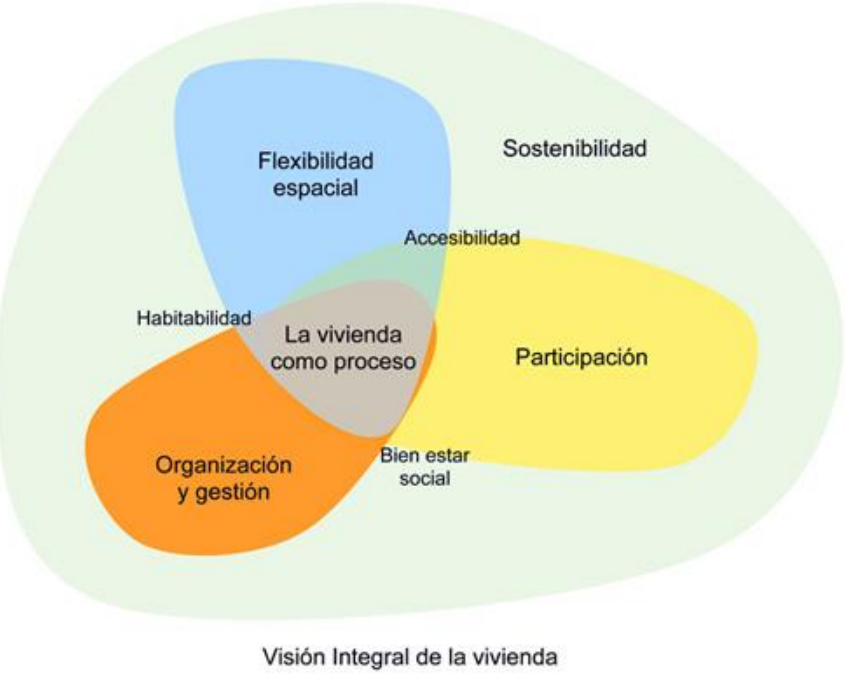

Figura 3. Esquema resumen de la vivienda como proceso y sus tres niveles. Fuente: Lapanadería.

La casa más o menos se estructura en torno a tres niveles: el nivel espacial-tipológico, el nivel social-participativo y el nivel organizativo y de gestión (figura 3 ). El primero de ellos, el nivel espacial-tipológico, es, tal vez, el más representativo de la idea inicial de casa más o menos. El objetivo en este ámbito es aportar a la vivienda la flexibilidad (cfr. Morales Soler, Alonso Mallén y Moreno Cruz 2012) que el CMI ha eliminado mediante la serialización de las personas y de sus deseos, y se centra sobre todo en la vertiente 
ALLEPUZ GARCÍA, Pablo (2016): "El estudio arquitectónico 'lapanadería' (20032013) desde la filosofía de Gilles Deleuze y Félix Guattari". Ángulo Recto. Revista de estudios sobre la ciudad como espacio plural, vol. 8, núm. 1-2, pp. 5-25. ISSN: 1989-4015.

http://dx.doi.org/10.5209/rev_ANRE.2016.v8.n1.53346

física, material, de la casa ( $\mathrm{y}$, llegado el caso, también del entorno). Para ello, primero, es necesario identificar las necesidades demandadas y, una vez tipificadas, establecer estrategias: en función del tipo de flexibilidad, pueden ser cualitativas, adaptables o elásticas; en función del modo en que conceden esa flexibilidad, pueden ser funcionales o formales (figura 4).

Estrategias aplicables tanto en viviendas aisladas como en agrupaciones de viviendas

Estrategias funcionales Estrategias formales

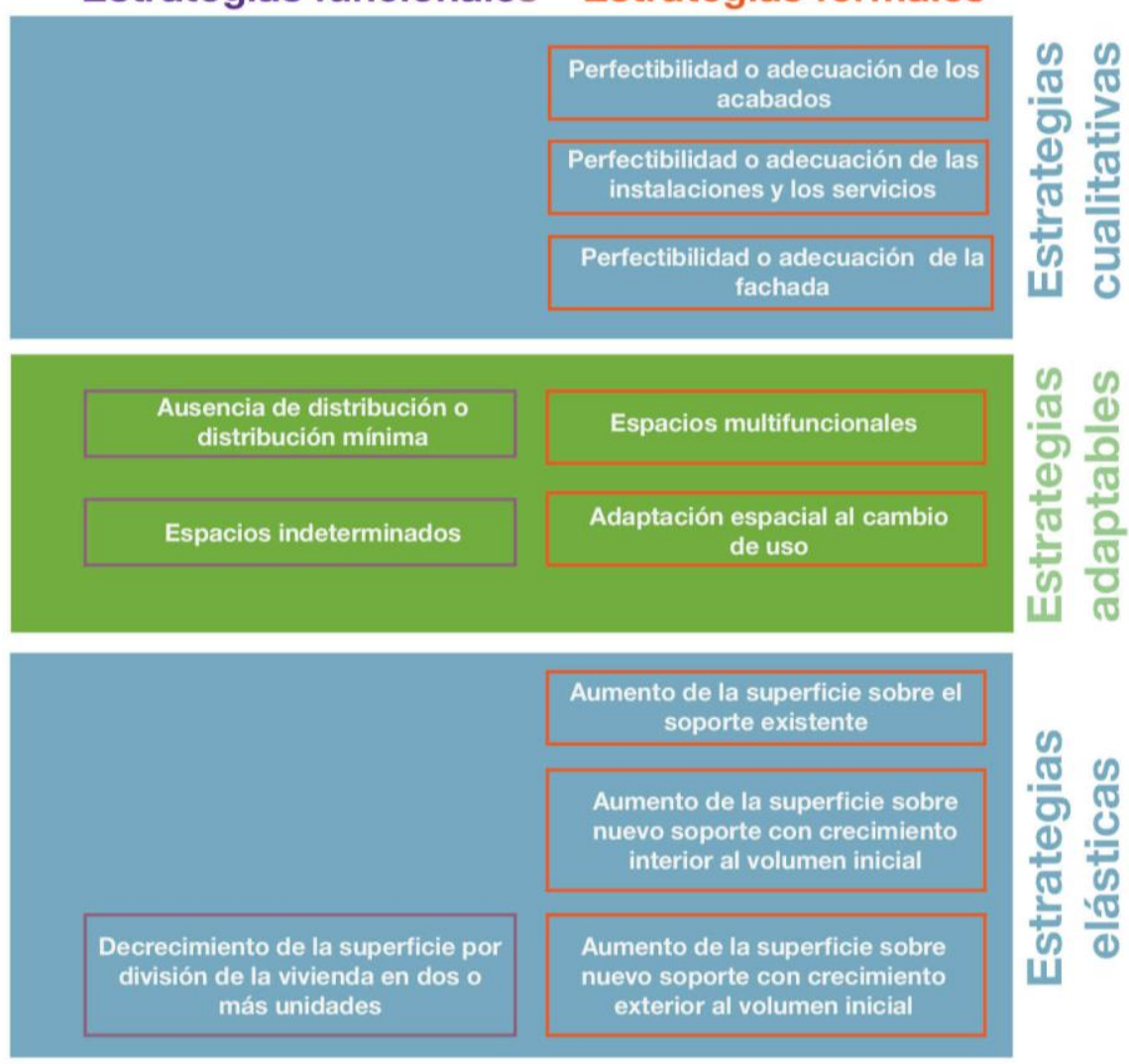

Estrategias aplicables exclusivamente en agrupaciones de viviendas

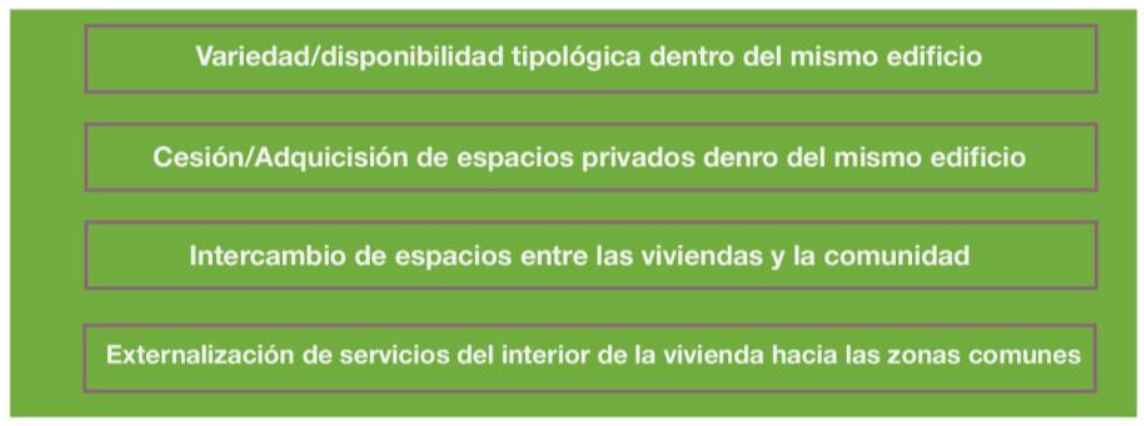

Figura 4. Tabla con las distintas estrategias para la vivienda individual y conjuntos de vivienda. Fuente: Lapanadería (2010: 63-64) retocada por Nando Dorrego. 
ALLEPUZ GARCÍA, Pablo (2016): "El estudio arquitectónico 'lapanadería' (20032013) desde la filosofía de Gilles Deleuze y Félix Guattari". Ángulo Recto. Revista de estudios sobre la ciudad como espacio plural, vol. 8, núm. 1-2, pp. 5-25. ISSN: 1989-4015.

http://dx.doi.org/10.5209/rev_ANRE.2016.v8.n1.53346

Una de las posibilidades teóricas de la casa más o menos es precisamente el desdoblamiento que Jorge Andrade estudió y aplicó en el contexto mexicano desde 1981 (vid. Lapanadería y Andrade 2010): obtener de un núcleo habitacional que daba cobijo a una familia completa nuevas células independientes, no solo habitaciones privadas, para que los hijos de la familia puedan desarrollar sus vidas más allá de la cohabitación familiar si, por ejemplo, debieran desplazarse por cuestiones laborales. La experiencia en otros países demuestra que la vivienda procesual, susceptible de modificaciones, supone un modelo útil para solventar cualquier tipo de problema (enfermedades, soledad...). Pero ello no surge de manera espontánea, sino que emana directamente del sentimiento de pertenencia a una comunidad que puede llevar a cabo de forma efectiva esos cambios. Es decir, la ecología social trabaja directamente sobre la mental, y viceversa, siempre que se haya generado previamente una comunidad implicada $y$, no menos importante, siempre que dichas prácticas estén amparadas bajo la normativa vigente en materia arquitectónica; lo cual, de momento, está lejos de ser una realidad en España.

La vertiente medioambiental de la casa más o menos no se encuentra tanto en los resultados prácticos -esto es, en el producto físico derivado de la intervención del arquitecto y de las sucesivas modificaciones de los habitantes- como en la propia coherencia implícita en el nuevo sistema. Mientras que en las prácticas habituales del CMI la casa se convierte, como todo lo demás, en un objeto desechable de muy corta vida, la casa más o menos permite reducir los costes, reutilizar sus elementos y reciclar los espacios; a la sazón, las tres "erres" de la manida ecología medioambiental.

En segundo lugar, el nivel social-participativo" "se hace inevitable e imprescindible como medio para definir la identidad individual y colectiva" (Lapanadería 2010: 111). Los actores que intervienen en los procesos participativos son las entidades políticas, el personal técnico y la ciudadanía, y, como vimos en la introducción, España adolece de un excesivo peso específico de los primeros en detrimento de las iniciativas populares que defienden Deleuze y Guattari.

Se necesita una inmensa reconstrucción de los mecanismos sociales para hacer frente a los estragos del CMI. Ahora bien, esta reconstrucción no depende tanto de reformas desde arriba, leyes, decretos, programas burocráticos, como de la promoción de prácticas

\footnotetext{
2 Enrique Ortiz Flores habla al respecto en una entrevista con lapanadería (vid. Lapanadería y Ortiz 2013b).
} 
ALLEPUZ GARCÍA, Pablo (2016): "El estudio arquitectónico 'lapanadería' (20032013) desde la filosofía de Gilles Deleuze y Félix Guattari". Ángulo Recto. Revista de estudios sobre la ciudad como espacio plural, vol. 8, núm. 1-2, pp. 5-25. ISSN: 1989-4015.

http://dx.doi.org/10.5209/rev_ANRE.2016.v8.n1.53346

innovadoras, la proliferación de experiencias alternativas, centradas en el respeto de la singularidad y en un trabajo permanente de producción de subjetividad, que se autonomicen al articularse convenientemente con el resto de la sociedad. (Guattari 1996: 61)

\begin{tabular}{|l|l|l|l|l|}
\hline Matriz de la & $\begin{array}{l}\text { Fase 1: } \\
\text { Diagnóstico y } \\
\text { prospectiva. }\end{array}$ & $\begin{array}{l}\text { Fase 2: } \\
\text { Planificación y } \\
\text { toma de } \\
\text { decisiones. }\end{array}$ & $\begin{array}{l}\text { Fase 3: } \\
\text { Diseño y } \\
\text { ejecución. }\end{array}$ & $\begin{array}{l}\text { Fase 4: } \\
\text { Retroalimenta- } \\
\text { ción y } \\
\text { seguimiento. }\end{array}$ \\
\hline Información & $\begin{array}{l}\text { La administración } \\
\text { inicia la acción }\end{array}$ & $\begin{array}{l}\text { La administración } \\
\text { planificación la acción }\end{array}$ & $\begin{array}{l}\text { La administración } \\
\text { implementa la acción }\end{array}$ & $\begin{array}{l}\text { La administración } \\
\text { mantiene la acción }\end{array}$ \\
\hline Consulta & $\begin{array}{l}\text { La administración } \\
\text { inicia la acción } \\
\text { después de consultar } \\
\text { a la comunidad }\end{array}$ & $\begin{array}{l}\text { La administración } \\
\text { planea la acción } \\
\text { después de consultar } \\
\text { a la comunidad }\end{array}$ & $\begin{array}{l}\text { La administración } \\
\text { implementa la acción } \\
\text { después de consultar } \\
\text { a la comunidad }\end{array}$ & $\begin{array}{l}\text { La administración } \\
\text { mantiene la acción } \\
\text { después de consultar a } \\
\text { la comunidad }\end{array}$ \\
\hline Cogestión & $\begin{array}{l}\text { La administración y la } \\
\text { comunidad inician la } \\
\text { acción conjuntamente }\end{array}$ & $\begin{array}{l}\text { La administración y la la } \\
\text { comunidad planifican } \\
\text { la acción } \\
\text { conjuntamente }\end{array}$ & $\begin{array}{l}\text { La administración y la } \\
\text { comunidad } \\
\text { implementan la } \\
\text { acción conjuntamente }\end{array}$ & $\begin{array}{l}\text { La administración y la } \\
\text { comunidad mantienen } \\
\text { la acción } \\
\text { conjuntamente }\end{array}$ \\
\hline Autogestión & $\begin{array}{l}\text { La comunidad inicia la } \\
\text { acción por su cuenta }\end{array}$ & $\begin{array}{l}\text { La comunidad } \\
\text { planifica por su } \\
\text { cuenta }\end{array}$ & $\begin{array}{l}\text { La comunidad } \\
\text { implementa la acción } \\
\text { por su cuenta }\end{array}$ & $\begin{array}{l}\text { La comunidad mantiene } \\
\text { la acción por su cuenta }\end{array}$ \\
\hline
\end{tabular}

Figura 5. Distintos niveles y ámbitos de participación ciudadana. Fuente: Lapanadería (2010: 106).

Así pues, lapanadería ofrece al habitante, al ciudadano, la posibilidad de participar en la gestión, en el diseño, en la construcción (o autoconstrucción) y en el uso y mantenimiento de la vivienda (figura 5); y todo ello se vehicula mediante metodologías participativas existentes, como "Investigación Acción Participativa" 0 "European Awareness Scenario Workshops", entre otras.

En tercer y último lugar, el nivel organizativo y de gestión se entiende como producción social del hábitat, atendiendo a "los procesos generadores de espacios habitables, componentes urbanos y viviendas, que se realizan bajo el control de autoproductores y otros agentes sociales que operan sin fines lucrativos" (Lapanadería 2010: 151). Los modelos propuestos, ya experimentados en el territorio español, son Aparcería Urbana, Autoconstrucción, Cooperativas, Cohousing, Okupación... En cierto sentido, este tercer nivel viene a solaparse con el anterior, y es que en realidad, como dice Guattari, no pueden ni deben entenderse unos sin otros, sino únicamente incardinados en una misma cartografía (cfr. Guattari y Rolnik 2006).

Todas estas ideas se pusieron en funcionamiento en 2005 dentro del proyecto "Alcalá 01" (figuras 6 y 7), en Alcalá de Guadaira (vid. Lapanadería 2008): un entramado urbano que, no obstante, dista del centro; es decir, el precio de suelo es asequible, el contexto 
ALLEPUZ GARCÍA, Pablo (2016): "El estudio arquitectónico 'lapanadería' (20032013) desde la filosofía de Gilles Deleuze y Félix Guattari". Ángulo Recto. Revista de estudios sobre la ciudad como espacio plural, vol. 8, núm. 1-2, pp. 5-25. ISSN: 1989-4015.

http://dx.doi.org/10.5209/rev_ANRE.2016.v8.n1.53346

social se muestra más abierto a las innovaciones y los beneficiarios fueron, fundamentalmente, jóvenes que accedían a su primera vivienda.

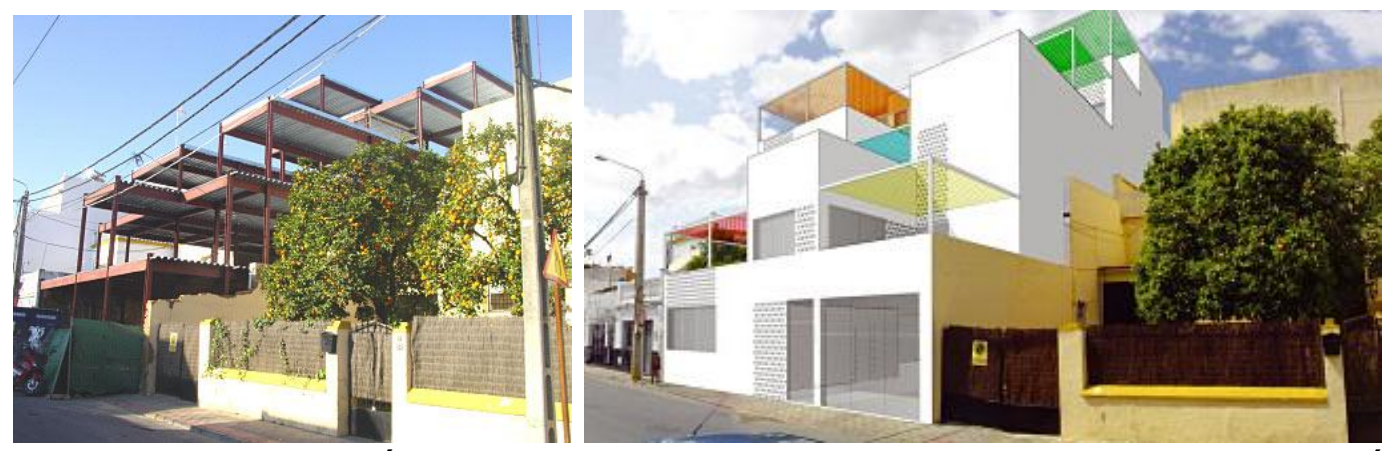

Figuras 6 y 7. "Alcalá 01", primera vivienda como proceso de lapanadería (Alcalá de Guadaira, Sevilla). Fuente: Lapanadería.

\section{Barrio más o menos}

Gran parte de las críticas que pudiéramos dirigir a la casa más o menos desde los preceptos guattarianos -a saber: aislamiento, escaso impacto social, flexibilidad relativa, etc.- se disipan en la multiplicación y configuración de un conjunto de casas más o menos. Ello consolidaría un barrio más o menos o barrio como proceso donde las relaciones vecinales comenzarían a entretejer una verdadera ecosofía: al compartir los recursos y aumentar el número de viviendas, los gastos disminuyen y se reparten; al gestionar los problemas y las decisiones en común, los vínculos entre los integrantes se fortalecen; al sentirse perteneciente a una comunidad, uno adquiere una mayor y mejor conciencia de sí mismo...

Un barrio constituido desde estos parámetros supone un rizoma en potencia; noción de sobra conocida que viene a sustituir la tradicional metáfora del árbol, binaria, dicotómica, lineal y jerárquica, por una lógica de la complejidad, de la multiplicidad y de la heterogeneidad, posibilitando cualquier tipo de conexión, rupturas y líneas de fuga (Deleuze y Guattari 2010: 9-29). Desde un punto bastante temprano de su producción, ambos omitían la separación tradicional entre lo privado y lo público, entre infraestructura y superestructura, poniéndolo todo en un mismo plano de importancia que podría, por tanto, ser atravesado transversalmente (Deleuze y Guattari 1973: 33; cfr. Martínez 2008: 56). Ello puede verse, por ejemplo, en la unión guattariana de análisis psicoanalítico y política, esquizoanálisis, que desemboca en grupos a un tiempo terapéuticos y políticos; estos serían la continuación del grupo-sujeto de Sartre, que se define como agente colectivo de enunciación por oposición al grupo-sometido, donde los miembros no expresan sus deseos, están 
ALLEPUZ GARCÍA, Pablo (2016): "El estudio arquitectónico 'lapanadería' (20032013) desde la filosofía de Gilles Deleuze y Félix Guattari". Ángulo Recto. Revista de estudios sobre la ciudad como espacio plural, vol. 8, núm. 1-2, pp. 5-25. ISSN: 1989-4015.

http://dx.doi.org/10.5209/rev_ANRE.2016.v8.n1.53346

cerrados al exterior y no tienen conciencia de sus límites (Martínez 2008: 12-13).

Tales barrios más o menos se han llevado a la práctica en proyectos como V.P.O. J5 (Vélez Rubio, Almería) y, en mayor medida, en Cerdanyola (figuras 8 y 9), finalista en EUROPAN 2011. En ambos casos, la configuración espacial de todo el barrio se diseña desde lo asociativo, desde las necesidades y las posibilidades de uso de los distintos integrantes de la comunidad.
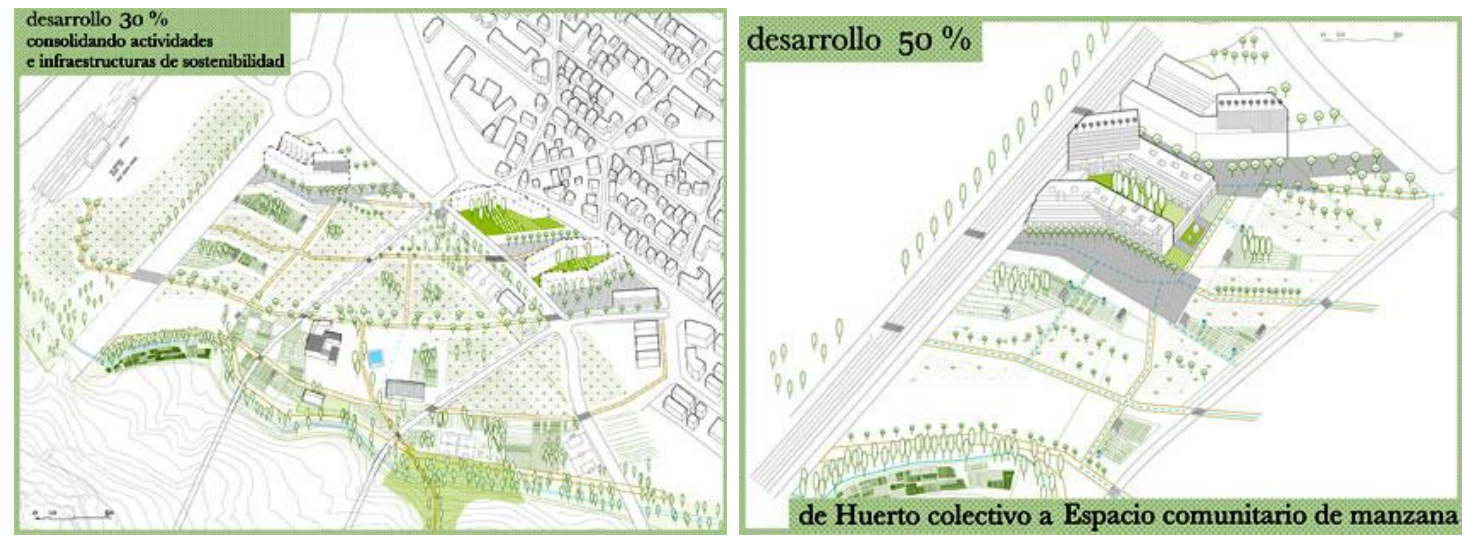

Figuras 8 y 9. Barrio como proceso (Cerdanyola, Barcelona).

Fuente: Lapanadería. 
ALLEPUZ GARCÍA, Pablo (2016): "El estudio arquitectónico 'lapanadería' (20032013) desde la filosofía de Gilles Deleuze y Félix Guattari". Ángulo Recto. Revista de estudios sobre la ciudad como espacio plural, vol. 8, núm. 1-2, pp. 5-25. ISSN: 1989-4015.

http://dx.doi.org/10.5209/rev_ANRE.2016.v8.n1.53346

\section{Más que una casa; ¿hacia una ciudad más o menos?}

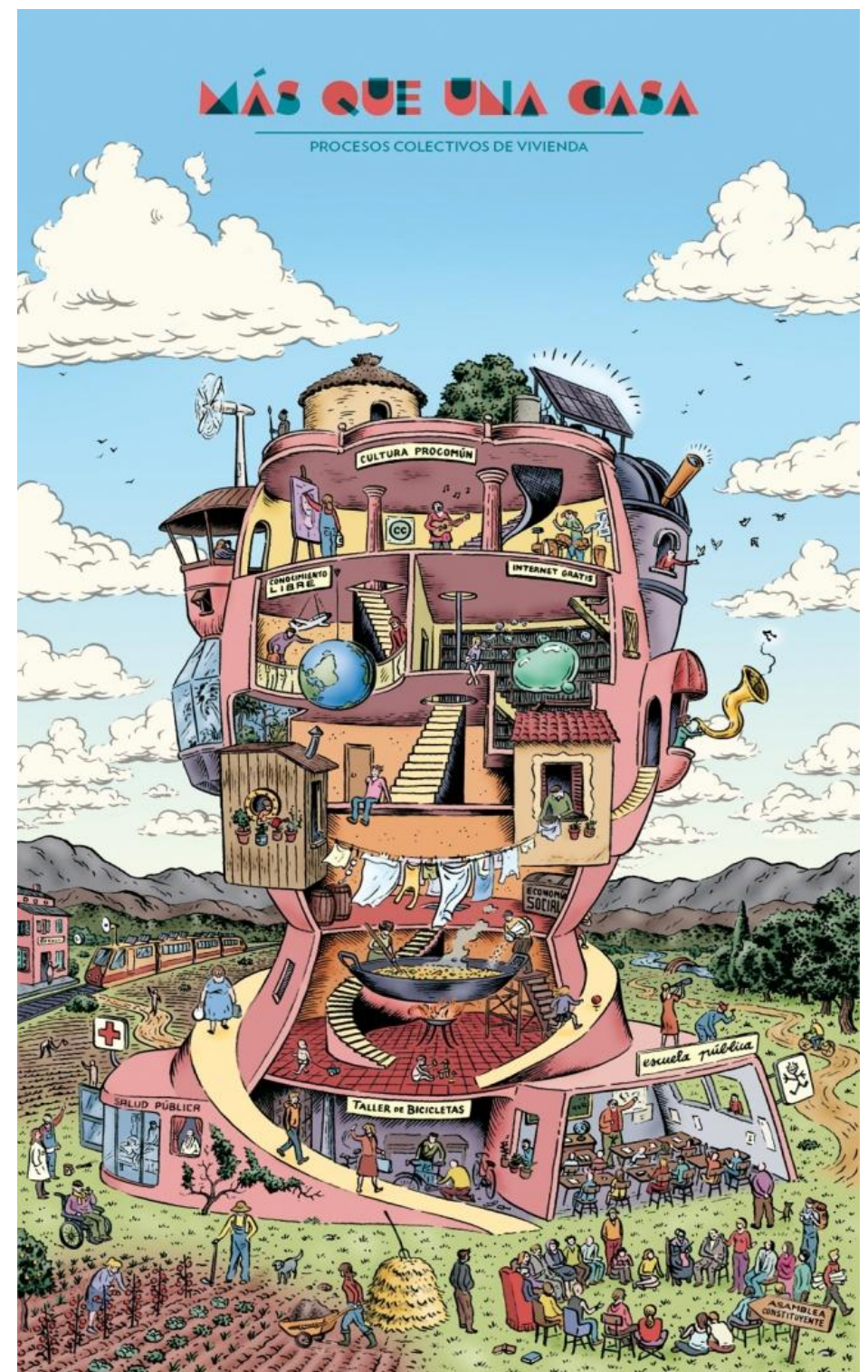

Figura 10. Imagen principal de la plataforma web Más que una casa.

Fuente: Lapanadería.

El derecho a la ciudad [expresión de Henri Lefebvre expuesta en su libro homónimo de la significativa fecha de 1968] es mucho más que la libertad individual de acceder a los recursos urbanos: se trata del derecho a cambiarnos a nosotros mismos cambiando la ciudad. Es, además, un derecho común antes que individual, ya que esta transformación depende inevitablemente del ejercicio de un poder colectivo para remodelar los procesos de urbanización. (Harvey 2008: 23) 
ALLEPUZ GARCÍA, Pablo (2016): "El estudio arquitectónico 'lapanadería' (20032013) desde la filosofía de Gilles Deleuze y Félix Guattari". Ángulo Recto. Revista de estudios sobre la ciudad como espacio plural, vol. 8, núm. 1-2, pp. 5-25. ISSN: 1989-4015.

http://dx.doi.org/10.5209/rev_ANRE.2016.v8.n1.53346

Después del barrio más o menos, todavía se podrían criticar algunas cuestiones con el mismo esquema que ya se había aplicado a la solitaria casa más o menos: aislamiento, escaso impacto social, flexibilidad relativa, etc., aunque se trate de una escala mayor. El siguiente paso, dentro de una evolución lógica, sería la expansión de estas ideas; y ello se realiza utilizando los propios medios del sistema que se intenta combatir y sus propias máquinas de progreso, de las que Deleuze y Guattari no renegaban: Más que una casa ${ }^{3}$ es una plataforma on-line que pretende funcionar como espacio para compartir y explicar todas aquellas posibilidades fuera de los discursos hegemónicos para organizarse, realizar y usar la vivienda.

El nivel social de la ecología se enfrenta hoy con el reto de superar la era mediática en que se ha convertido la actual fase del desarrollo capitalista en dirección a una era post-mediática en la que las potencialidades implícitas en la actual revolución informática se desplieguen gestionadas por una serie coordinada de grupos-sujetos dando lugar a un proceso de singularización creciente. (Martínez 2008: 169)

Tal vez, por tanto, la producción de subjetividad del ámbito de la vivienda, y del ámbito del barrio, deba desembocar en una producción en el ámbito de la ciudad para, posteriormente, establecerse como modelo a escala global, derrocando por fin al CMI; curiosamente, en una sucesión de etapas similar a la de los CIAM: vivienda, barrio, ciudad, hábitat, hábitat humano. Pero, ¿bajo qué parámetros se constituiría este modelo?

El paradigma cientifista dominante tanto en el psicoanálisis como en el marxismo Guattari lo sustituye por un paradigma ético-estético, ético porque se basa en la responsabilidad por las consecuencias que se derivan de nuestras acciones, y estético porque tiene un aspecto creativo, de generación de elementos inéditos, y además se refiere a la sensibilidad, a los sentidos. (Martínez 2008: 174)

Ya hemos tratado lo ético. En cuanto a lo estético, un capítulo interesante en Deleuze y Guattari es el concepto de rostridad (visageité) (2010: 173-194), que aplican entre otros ejemplos a la pintura de Francis Bacon y a las películas de Griffith y Eisenstein:

Los rostros concretos son el producto de una máquina abstracta de rostridad [...]. El rostro es el elemento clave de la individuación subjetiva y por ello sobredetermina el conjunto del cuerpo de tal

3 Puede consultarse en http://masqueunacasa.org/es. Véase también la presentación de Alonso y Morales (2012). 
ALLEPUZ GARCÍA, Pablo (2016): "El estudio arquitectónico 'lapanadería' (20032013) desde la filosofía de Gilles Deleuze y Félix Guattari". Ángulo Recto. Revista de estudios sobre la ciudad como espacio plural, vol. 8, núm. 1-2, pp. 5-25. ISSN: 1989-4015.

http://dx.doi.org/10.5209/rev_ANRE.2016.v8.n1.53346

manera que se produce un salto del sistema cuerpo-cabeza al sistema rostro [...]. El sistema rostro, pues, es el resultado de una producción social que personaliza y normaliza los individuos, espiritualizándolos y desnaturalizándolos, de manera tal que podremos decir, que "el rostro es una política". (Martínez 2008: 69)

Así pues, si la ciudad era cuerpo sin órganos la casa bien puede ser rostro: casa-rostro de la ciudad-cuerpo, pues en aquella se da la máxima (des-/re-)territorialización de esta. Llegados a este punto, se cierra el círculo: interprétese de nuevo la portada del proyecto más que una casa (figura 10) a la luz de estas sentencias y se podrá encontrar lo artístico, por lo demás obvio, pero también la individuación, el sistema, la producción social, la organización, la macro y la micropolítica...; es decir, todos los conceptos enumerados y aplicados a lo largo de estas páginas.

De igual manera que el espacio se hace familiar mediante mecanismos sociales que marcan a través del sistema rostro lo aceptable, el tiempo humano también está construido mediante dispositivos concretos de semiotización que tiende a establecer pautas repetitivas para conjurar el caos y establecer nichos de familiaridad en los que cada grupo o incluso cada individuo se pueda reconocer. Estos dispositivos repetitivos son los ritornelos que actúan como "ritmos básicos de la temporalización". (Martínez 2008: 75)

\section{5. ¿Más, o menos? Últimas consideraciones}

A pesar de todas las aportaciones comentadas en los apartados anteriores, podríamos plantear algunas críticas menores a la propuesta de lapanadería. En primer lugar, desde un punto de vista teórico, la vivienda como proceso parte de una contradicción en sus términos: en sentido estricto, es ilógico plantear con los materiales y técnicas del presente una estructura para el futuro, pues desconocemos por completo cómo será la casa del futuro y corremos el riesgo de caer de nuevo en los errores del pasado. Igualmente, las personas no habitan más o menos y la casa más o menos es un producto terminado, aunque se trate de una terminación más o menos precaria o provisional. En cualquier caso, ya supone un avance considerable respecto a la inmovilidad de los discursos pretendidamente eternos del CMI (cfr. Deleuze y Guattari 1973: 39) $y$, desde un punto de vista práctico, resulta una gran herramienta para el corto y medio plazo.

Dentro de este contexto de indefinición, la casa más o menos afrontará otro problema: el choque frontal contra la globalización. La transformación de las necesidades de la persona verá su correlato en la transformación de la casa, pero sigue siendo una transformación 
ALLEPUZ GARCÍA, Pablo (2016): "El estudio arquitectónico 'lapanadería' (20032013) desde la filosofía de Gilles Deleuze y Félix Guattari". Ángulo Recto. Revista de estudios sobre la ciudad como espacio plural, vol. 8, núm. 1-2, pp. 5-25. ISSN: 1989-4015.

http://dx.doi.org/10.5209/rev_ANRE.2016.v8.n1.53346

atada al suelo como los duelistas de Goya; el mundo globalizado, de libre mercado, exige adaptaciones más allá del propio terruño, esto es, movimientos de localización, un cierto grado de nomadismo. La vivienda como proceso se adapta a las necesidades que el habitante pueda tener dentro de la casa, pero ¿qué ocurre si sus necesidades lo llevan a varios cientos de kilómetros de su ubicación original? ¿Qué ocurriría con esa casa que empezó siendo más o menos y que al cabo del tiempo terminó siendo una casa totalmente subjetivizada a la medida de sus habitantes? ¿Quién compraría y se haría cargo de una casa desde un nivel tal de subjetivación, con todo el esfuerzo de adaptación que conllevaría? ¿No exigiría ello, en el sentido de la sostenibilidad, una inversión y un desperdicio de recursos mayor que en una casa habitual? Existe, en suma, todo un espectro de casuísticas similares que deberían contemplarse en el proyecto general y que, en cambio, se han omitido.

De entre los ámbitos de la producción estética, la arquitectura es, con toda probabilidad, el más dependiente de los factores normativos y, sobre todo, económicos. La creatividad, por tanto, se ve siempre coartada por la trascendencia de las acciones experimentales, con dos principales consecuencias: por una parte, aquellas suelen ser limitadas y marginales y, por otra, la producción de subjetividad estética está reservada a aquellos que pueden permitírselo, quedando las minorías fuera del discurso de reterritorialización; incluso, cabe suponer, en la propuesta de lapanadería. Es decir, habría que preguntarse por la incidencia de estas minorías en el proceso de la casa o del barrio más o menos, o, por decirlo con Deleuze, de qué manera pueden la vivienda o el barrio devenir-mujer, devenir-niño, devenir-animal, en suma, devenirmolecular.

La propuesta de lapanadería, y en general todos los planteamientos arquitectónicos que anteponen lo procesual a los productos de pretensiones eternas, comportan por último un importante riesgo. El sistema capitalista actual ha extendido sus raíces y raicillas (Deleuze y Guattari 2010: 9-13) de manera tan extraordinaria precisamente debido a su habilidad de introducir en los individuos necesidades innecesarias. Hasta ahora, el inmovilismo en el ámbito de la arquitectura doméstica había mantenido la amenaza capitalista dentro de la estructura del hogar pero no tanto en la propia estructura doméstica (burbujas inmobiliarias aparte, por supuesto). Sin embargo, tales planteamientos, por más que su concepción tuviera lugar fuera del sistema e incluso contra el sistema, se arriesgan a ser fagocitados y utilizados por el propio sistema; ese es, en cualquier caso, el terrible potencial del capitalismo, capaz de absorber todo tipo de tendencia y ponerla en 
ALLEPUZ GARCÍA, Pablo (2016): "El estudio arquitectónico 'lapanadería' (20032013) desde la filosofía de Gilles Deleuze y Félix Guattari". Ángulo Recto. Revista de estudios sobre la ciudad como espacio plural, vol. 8, núm. 1-2, pp. 5-25. ISSN: 1989-4015.

http://dx.doi.org/10.5209/rev_ANRE.2016.v8.n1.53346

funcionamiento bajo sus parámetros y mecanismos mercantiles (Guattari 1996: 13), generando una dependencia más en los habitantes.

La pregunta que surge entonces es si es suficiente, desde un punto de vista teórico, la acción subjetiva para guiar una práctica que alcanza un alto grado de implicación social. ¿Estaría asegurado que la proyección de una determinada subjetividad aseguraría un escenario suficiente para la vida completa? La filosofía se ha interrogado en las últimas décadas sobre las características del sujeto contemporáneo, el dúo Heidegger-Levinas estaría en la base de esta consideración y sus conclusiones -como nos ha demostrado Dal Co o Teyssot- son absolutamente relevantes para la arquitectura. Pero ha sido Giorgio Agamben, tomando como base la denuncia de Debord, el que nos ha hecho ver que la expropiación del lenguaje como experiencia sujeto, ha venido acompañada de la expropiación de nuestra experiencia habitativa. (Moreno García 2000-2001: 150)

Aunque también podría suceder en el sentido contrario: si la obra abierta de Eco y las reflexiones paralelas de Roland Barthes o Michel Foucault condujeron primero a la participación del lector en la construcción de la obra y, consecuentemente, a la muerte del autor, ¿conducen las propuestas de lapanadería, y otras similares, a una muerte del arquitecto o, cuanto menos, a una arquitectura sin arquitectos, en la que por fin el habitante adquiriera un papel protagonista...?

En relación con la arquitectura Guattari plantea cómo es posible practicarla todavía cuando su objeto ha estallado. Lo cual supone que hay que recomponer la enunciación arquitectónica e incluso el oficio de arquitecto. Este no se puede limitar a ser un plástico de formas construidas sino que tiene que ser un revelador de los deseos vehiculados por el espacio. En este sentido el concepto arquitectónico tiene dos componentes: una, discursiva, del orden del percepto y otra, no-discursiva, del orden del afecto. Lo que definiría hoy al arquitecto es su capacidad de captar los afectos de la enunciación espacial, que puedan ser capaces de funcionar como un catalizador de las semióticas espaciales capaces de hacernos salir de nosotros mismos y de ponernos en contacto con campos posibles inéditos. La arquitectura tendrá que ser capaz de producir un enriquecimiento existencial a través de la generación de un acontecimiento que pueda resingularizar el deseo y los valores en una coyuntura histórica dada. (Martínez 2008: 185) 
ALLEPUZ GARCÍA, Pablo (2016): "El estudio arquitectónico 'lapanadería' (20032013) desde la filosofía de Gilles Deleuze y Félix Guattari". Ángulo Recto. Revista de estudios sobre la ciudad como espacio plural, vol. 8, núm. 1-2, pp. 5-25. ISSN: 1989-4015.

http://dx.doi.org/10.5209/rev_ANRE.2016.v8.n1.53346

\section{Bibliografía}

ALONSO, Rubén; y MORALES, Eva (2012): "Plataforma web de procesos colectivos de vivienda" [en línea], en $X X$ Seminario de Arquitectura y Compromiso Social "El cooperativismo de vivienda en cesión de uso: conceptos, reflexiones, experiencias y propuestas". En: https://vimeo.com/43313365 [Consulta: 27/08/2016].

ARAVENA, Alejandro (2008): "(Utopías necesarias). Martes, 2 de octubre de 2007. 19:45 h. V. O.", en Juan Herreros (coord.), Vivienda y espacio doméstico en el siglo XXI, pp. 114-123. Madrid: La Casa Encendida.

ARAVENA, Alejandro; y IACOBELLI, Andrés (2012): ELEMENTAL: Manual de vivienda incremental y diseño participativo. Ostfildern: Harje Cantz.

DELEUZE, Gilles (2009): Francis Bacon. Lógica de la sensación. Madrid: Arena Libros.

DELEUZE, Gilles; y GUATTARI, Félix (1973): El Antiedipo. Capitalismo y esquizofrenia. Barcelona: Barral.

DELEUZE, Gilles; y GUATTARI, Félix (1997): ¿Qué es la filosofía? Barcelona: Anagrama.

DELEUZE, Gilles; y GUATTARI, Félix (2010): Mil Mesetas. Capitalismo y esquizofrenia. Valencia: Pre-Textos.

GARCÍA VÁZQUEZ, Carlos (2004): Ciudad hojaldre. Visiones urbanas del siglo XXI. Barcelona: Gustavo Gili.

GUATTARI, Félix (1996): Las tres ecologías. Valencia: Pre-Textos.

GUATTARI, Félix; y ROLNIK, Suely (2006): Micropolítica. Cartografías del deseo. Madrid: Traficantes de Sueños.

HARVEY, David (2008): "El derecho a la ciudad". New Left Review, núm. 53, pp. 23-39.

HEIDEGGER, Martin (2007): Filosofía, ciencia y técnica. Santiago de Chile: Editorial Universitaria.

LAPANADERÍA (2008): "Casa más o menos. Proyecto Alcalá 01. Alcalá de Guadaira, Sevilla". On diseño, núm. 295, pp. 230-235. En: https://depan.s3.amazonaws.com/pan file/file/807/on diseno n295 casa mas menos.pdf [Consulta: 27/08/2016[.

LAPANADERÍA (2010): Proyecto de investigación "casa más o menos: la vivienda como proceso" [en línea]. Sevilla: Subvenciones para actividades de investigación en materia de vivienda y arquitectura / Consejería de Vivienda y Ordenación del Territorio de la Junta de Andalucía. En: https://ws147.juntadeandalucia.es/obraspublicasyvivienda/dav webviv/Proyectos\%20de\%20Investigacion/38\%20Casa\%20mas $\% 200 \% 20$ menos/1\%20PROYECTO\%20DE\%20INVESTIGACI\%C 3\%93N.pdf [Consulta: 27/08/2016]. 
ALLEPUZ GARCÍA, Pablo (2016): "El estudio arquitectónico 'lapanadería' (20032013) desde la filosofía de Gilles Deleuze y Félix Guattari". Ángulo Recto. Revista de estudios sobre la ciudad como espacio plural, vol. 8, núm. 1-2, pp. 5-25. ISSN: $1989-4015$.

http://dx.doi.org/10.5209/rev_ANRE.2016.v8.n1.53346

LAPANADERÍA (2012): Breve dossier [en línea]. En: https://depan.s3.amazonaws.com/pan file/file/961/lapanaderia dossier feb 2012.pdf [Consulta: 27/08/2016].

LAPANADERÍA (2013a): Lapanadería: arquitectura y diseño [en línea]. En: http://www.despachodepan.com [Consulta: 27/08/2016].

LAPANADERÍA (2013b): "Procesos" [en línea], en Lapanadería: arquitectura y diseño. En: http://www.despachodepan.com/procesos/ [Consulta: 27/08/2016].

LAPANADERÍA; y ANDRADE, Jorge (2010): Entrevista a Jorge Andrade: La vivienda social en México [en línea]. En: https://www.youtube.com/watch?v=mYd3VwEholg [Consulta: 27/08/2016].

LAPANADERÍA; y LORENZO, Pedro (2010): Entrevista a Pedro Lorenzo: La Cooperativa [en línea]. En: https://www.youtube.com/watch?v=eZOgJs40uVE [Consulta: 27/08/2016].

LAPANADERÍA; y ORTIZ (2013a): Entrevista a Enrique Ortiz: La vivienda como objeto económico, la vivienda como proceso social [en línea]. En: https://www.youtube.com/watch?v=yDfL]xjuK-4 [Consulta: 27/08/2016].

LAPANADERÍA; y ORTIZ (2013b): Entrevista a Enrique Ortiz: Sobre la participación [en línea]. En: https://www.youtube.com/watch?v=9qD3-nMnMSs [Consulta: 27/08/2016].

MARTÍNEZ, Francisco José (2008): Hacia una era post-mediática. Ontología, política y ecología en la obra de Félix Guattari. Madrid: Montesinos.

MORALES SOLER, Eva; ALONSO MALLÉN, Rubén; y MORENO CRUZ, Esperanza (2012): "La vivienda como proceso. Estrategias de flexibilidad". Hábitat y Sociedad, núm. 4, pp. 33-54.

MORENO GARCÍA, José Ramón (2000-2001): "Mundo, entorno, proyecto: umbrales y pliegues". Revista de Historia y Teoría de la Arquitectura, núm. 2-3, pp. 143-152.

MORENO RANGEL, David (2002-2003): "El problema del habitar en el siglo XXI". Revista de Historia y Teoría de la Arquitectura, núm. 4-5, pp. 97-130.

MUÑOZ, Francesc (2008): Urbanalización. Paisajes comunes, lugares globales. Barcelona: Gustavo Gili.

PARDO, José Luis (2014): A propósito de Deleuze. Valencia: PreTextos. 
ALLEPUZ GARCÍA, Pablo (2016): "El estudio arquitectónico 'lapanadería' (20032013) desde la filosofía de Gilles Deleuze y Félix Guattari". Ángulo Recto. Revista de estudios sobre la ciudad como espacio plural, vol. 8, núm. 1-2, pp. 5-25. ISSN: 1989-4015.

http://dx.doi.org/10.5209/rev_ANRE.2016.v8.n1.53346

PARRA BAÑóN, José Joaquín (2004-2005): "Ciudad hojaldre. Visiones urbanas del siglo XXI: La incierta ciudad exfoliable". Revista de Historia y Teoría de la Arquitectura, núm. 6-7, pp. 395-398.

PÉREZ DE LAMA, José (2002-2003): "Flujos antagonistas / geografías de la multitud". Revista de Historia y Teoría de la Arquitectura, núms. 4-5, pp. 187-225. 